Available online at www.sciencedirect.com

www.cya.unam.mx/index.php/cya

Contaduría y Administración 60 (S2) 128-146

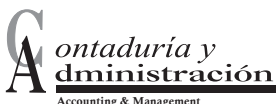

www.contaduriayadministracionunam.mx

\title{
La crisis financiera internacional de 2008 y algunos de sus efectos económicos sobre México
}

\author{
The international financial crisis of 2008 and some \\ of its economic effects on Mexico
}

\author{
Gloria de la Luz Juárez ${ }^{\mathrm{a}}$, Alfredo Sánchez Daza \\ Jesús Zurita González ${ }^{\text {a,* }}$
}

${ }^{a}$ Universidad Autónoma Metropolitana, Unidad Azcaptzalco, México

Recibido el 27 de junio de 2014; aceptado el 05 de octubre de 2015

Disponible en internet el 20 de noviembre de 2015

\section{Resumen}

La crisis financiera internacional de 2008 fue en su mayoría producto de la crisis de los derivados de hipotecas de baja calidad en EE.UU., que repercutió de forma negativa en la economía mundial. Los efectos adversos sobre la economía mexicana se manifestaron a través del canal de las exportaciones, afectando la producción industrial y manufacturera en particular en los estados del norte de México. Pruebas de causalidad de Granger muestran que la producción industrial de EE.UU. causa (en el sentido de Granger) la producción manufacturera de México. Se plantea un modelo econométrico VAR (de vectores autorregresivos) que incluye la producción industrial de EE.UU. como variable exógena, el cual indica que el tipo de cambio real tiene un efecto negativo sobre la producción manu-

\footnotetext{
* Autor para correspondecia:

Correo electrónico: jesus.zurita.gonzalez@gmail.com (J. Zurita González)

La revisión por pares es responsabilidad de la Universidad Nacional Autónoma de México.

http://dx.doi.org/10.1016/j.cya.2015.09.011

0186-1042/Derechos Reservados (C) 2015 Universidad Nacional Autónoma de México, Facultad de Contaduría y Admi- nistración. Este es un artículo de acceso abierto distribuido bajo los términos de la Licencia Creative Commons CC BY-NC-ND 4.0.
} 
facturera mexicana en el corto plazo y que las políticas monetaria y fiscal tienen un efecto poco importante sobre dicha producción, quizá porque estas políticas han sido procíclicas en el pasado. Para contrarrestar los efectos negativos de las crisis económicas internacionales, México necesita un mercado interno más desarrollado y poder implementar políticas fiscal y monetaria contracíclicas.

Derechos Reservados (C) 2015 Universidad Nacional Autónoma de México, Facultad de Contaduría y Administración.

Este es un artículo de acceso abierto distribuido bajo los términos de la Licencia Creative Commons CC BY-NC-ND 4.0.

Palabras clave: Crisis financiera internacional; Derivados de hipotecas de baja calidad; Producción industrial; Causalidad de Granger; Mercado interno.

Clasificación JEL: F3, F4, F6.

\section{Abstract}

The international financial crisis of 2008 due mainly to the subprime mortgage derivatives crisis in the United States but with world economic effects, had a significant negative repercussion on the Mexican economy mostly trough the real channel of the Mexican exports, which was reflected upon manufacturing and industrial production, particularly in the Northern states of Mexico. Granger causality tests show that the industrial production of the United States causes Mexican manufacturing production. A VAR econometric model which has U.S. industrial production as an exogenous variable indicates that the real exchange rate has a negative short-run effect on Mexican manufacturing production, and that fiscal and monetary policies have a small effect on manufacturing output. Moreover, it shows that these policies have been pro-cyclical in the past. To counter negative effects from international economic crises Mexico needs to develop a stronger domestic market and be able to forge counter cyclical monetary and fiscal policies.

All Rights Reserved (C) 2015 Universidad Nacional Autónoma de México, Facultad de Contaduría y Administración.

This is an open access item distributed under the Creative Commons CC License BY-NCND 4.0.

Keywords: International financial crisis; Subprime mortgage derivatives; Industrial production; Granger causality; Domestic market

JEL Classification: F3, F4, F6.

\section{Introducción}

La crisis financiera internacional de 2008 tuvo su origen en el problema de las hipotecas subprime de EE.UU. y se extendió después, afectando no sólo a toda la economía de ese país, sino también a las de otras naciones, de manera principal, miembros de la Unión Europea. En algunos casos, la contracción de la actividad económica y el empleo ha tenido incluso una mayor virulencia con respecto a lo 
que ha ocurrido en el país de origen. Por su parte, aunque en un principio las economías emergentes casi no fueron afectadas, con el paso del tiempo, las repercusiones financieras y reales de la debacle las alcanzaron.

Esta crisis, la mayor desde la Gran Depresión de 1929, ha marcado el desempeño de la economía mundial durante los últimos cinco años; en este periodo ésta creció seis puntos porcentuales menos en términos reales que si lo hubiera hecho a la tasa promedio de los veintiocho años previos: durante 1980-2007, la economía del orbe creció a 3\% promedio anual, mientras que a partir de 2008 y hasta 2012, lo hizo a 1.8\%, según cifras del Fondo Monetario Internacional (base de datos del Panorama Económico Mundial del Fondo Monetario Internacional, abril de 2012).

En los primeros años del siglo XXI, la evolución macroeconómica de EE.UU. implicaba un déficit considerable de cuenta corriente, el cual como porcentaje del PIB, alcanzó 6\% en 2006. En un marco de tasas de interés bajas y abundante liquidez, propiciado por la propia política monetaria de EE.UU., tales déficit se financiaban sin dificultad con entradas de capital. Al mismo tiempo, ocurría en el mundo, y de manera particular en EE.UU., un proceso de profundización de la innovación financiera que se retroalimentaba de la expansión del crédito. Éste impulsaba la innovación financiera, que buscaba incrementar la rentabilidad de los recursos financieros que podían obtenerse con relativa facilidad. La innovación financiera, a su vez, permitía mayor disponibilidad de recursos para los agentes económicos.

Visto con la perspectiva de lo pasado, parece claro que las autoridades monetarias y financieras de EE.UU. no consideraban al déficit de cuenta corriente como un problema: la productividad había estado aumentando y las empresas de ese país estaban obteniendo utilidades importantes, sobre todo las del sector financiero. Ambas condiciones parecían demostrar que el déficit de cuenta corriente era consecuencia de un fenómeno de equilibrio. Mayores entradas de capital por la mayor productividad y utilidades de las empresas tenían que ocasionar déficit de cuenta corriente como contrapartida. Una economía del tamaño de la estadounidense, con el sistema financiero más dinámico y desarrollado del mundo, podía acomodar fácil dicho déficit.

Pero la expansión acelerada del crédito y la innovación financiera tenían un lado oscuro que las autoridades financieras y monetarias de EE.UU. relegaban por considerarlo no importante: se retroalimentaban porque había una regulación laxa ante las prácticas, en varios casos muy dudosas, que prevalecían en el sistema financiero. Las instituciones financieras, con operaciones over the counter, no reguladas, se expandieron de forma significativa porque era donde se ubicaban las grandes ganancias. Las mismas instituciones bancarias y crediticias sujetas a regulación buscaron la manera de crear otras instituciones (shadow banking) que no tu- 
vieran que sujetarse a la regulación bancaria más estricta (Rodríguez, et. al., 2008).

El problema de información asimétrica, usual en el sistema financiero' ${ }^{1}$, ocasionó que muchos inversionistas no se dieran cuenta de los riesgos de activos respaldados por productos surgidos de la innovación financiera. Invertían, en cierto sentido, a ciegas, y estaban felices con sus elevados rendimientos en el contexto de crédito fácil y bursatilización inmediata. En el marco de la jauja financiera, las instituciones bancarias en EE.UU. operaron sin el debido cuidado, exacerbando el problema de riesgo moral. No se tenía cuidado al contabilizar los créditos de dudosa calidad, de alto riesgo.

Se distorsionaron los incentivos. Ello impidió actuar de manera prudente, cuidando los activos de las instituciones financieras. Con la bursatilización inmediata de la cartera de crédito, además, al vender los derivados, se deshacían del riesgo crediticio y evitaban pérdidas a futuro.

Podían bursatilizar hipotecas de alto riesgo sin demasiado cuidado. Se ocultaba el alto riesgo en los derivados financieros y se trasladaba hacia otros agentes económicos, con frecuencia, otras instituciones financieras, lo que después se convirtió en un serio problema de contagio. Se trataba de generar la mayor cantidad de hipotecas para poder bursatilizarlas y deshacerse del riesgo, casi con independencia de la capacidad de pago de los acreditados. Muchas instituciones financieras empezaron a operar con crédito de muy corto plazo y tenían pasivos importantes fuera de sus balances, lo que creó una enorme vulnerabilidad sistémica.

La crisis se produjo cuando empezaron a caer los precios de los bienes raíces. Al incumplir los deudores con los pagos de sus hipotecas, en su mayoría, de baja calidad crediticia (subprime), el castillo de naipes empezó a derrumbarse (Lane, 2013). El contagio se hizo presente. Al no conocerse la magnitud de los pasivos netos fuera del balance de las instituciones financieras, se paralizó el crédito, y esto provocó una crisis sistémica (CEPAL, 2009).

El problema, que parecía circunscribirse a EE.UU., se extendió rápido a otras partes del mundo (Chinn, 2012), principalmente, a otras economías avanzadas, así como hacia las emergentes (Eichengreen, 2012), en particular, México. Para atender la situación económica y financiera crítica, las economías avanzadas, principalmente EE.UU. y la Unión Europea, han recurrido a medidas nunca antes vistas como el denominado quantitative easing en la política monetaria (Nayak, 2013), otorgando facilidades de crédito y crédito en cantidades extraordinarias a las instituciones financieras para evitar una crisis de liquidez y posterior paralización de la economía (Tanzi, 2011).

\footnotetext{
${ }^{1}$ Los inversionistas no contaban con información suficiente que les permitiera conocer el elevado riesgo que implicaba adquirir activos financieros respaldados por hipotecas subprime.
} 
Los efectos sobre la economía mexicana fueron más significativos en los estados del norte del país, que registran mayor vinculación con la economía estadounidense. Por ello, se dedica una sección a esto.

En la segunda sección, se examina la relación estrecha entre la producción industrial de México y la de EE.UU., que se refleja en el comercio entre ambos países. Se comentan también algunos efectos sobre el sector financiero.

En la tercera sección, se reportan los efectos de la crisis en la producción manufacturera de la región norte de México, la más afectada por su mayor vinculación con la economía de nuestro principal socio comercial.

En la cuarta sección, se describen los resultados de un modelo VAR de corto plazo de la producción manufacturera mexicana, que incorpora los efectos de la producción industrial estadounidense como variable exógena.

En la sección quinta, se presentan algunos comentarios que recalcan la importancia para la economía mexicana de contar con fuentes internas de crecimiento para reducir su dependencia de la economía estadounidense en la actualidad.

En este trabajo, se examinan los efectos de esta crisis sobre la economía mexicana hasta 2012, resaltando sus consecuencias para la industria y el comercio internacional. Aunque las repercusiones de esta crisis son mundiales y han sido en particular profundas para algunos países de la Unión Europea, como Grecia, Portugal, España, Italia e Irlanda, el trabajo se ocupa del caso mexicano.

En general, el mecanismo de transmisión de la crisis financiera de EE.UU. hacia otros países ocurrió a través tanto del propio sector financiero como del sector real. Pero en el caso mexicano, es claro que los efectos de esta crisis se transmitieron a través del sector real, en particular, a través del estrecho vínculo comercial entre las dos economías, asociado a la complementariedad en la producción industrial.

\section{La vinculación de la economía mexicana a la estadounidense}

A partir al menos de la segunda mitad del siglo XX, la economía mexicana ha registrado una vinculación significativa con la estadounidense debido a la vecindad geográfica. El gran tamaño y la cercanía del mercado estadounidense lo hacen muy atractivo para las exportaciones mexicanas (Hernández, 2010), aunque ello también genera dependencia por la vía del comercio entre los dos países: si la economía estadounidense no anda bien, afecta de forma negativa las exportaciones de México y, como consecuencia, también su crecimiento, y crea una situación que, sobre todo en el corto plazo, es más difícil para México que para otros países (Vidal et. al., 2011). El resto de las economías grandes de América Latina, quizá con la excepción de Chile, se ven más afectadas por el desempeño de China y de la Unión Europea (Ocampo, 2009). 
Las relaciones económicas de dependencia, con la evolución de la economía mexicana dependiendo del desempeño de la estadounidense, se han ido acentuando no tanto por el distinto grado de desarrollo, sino por la carencia de fuentes internas de crecimiento y la relación comercial más estrecha con nuestro vecino del norte. A partir del TLCAN, los vínculos se apretaron aún más, e incluso ahora se habla de la sincronía que existe en el comportamiento de las dos economías, en particular, en el sector industrial.

Aunque aquí no se comenta el efecto de las remesas provenientes de EE.UU., la desaceleración económica de ese país influyó también para que éstas se redujeran y afectaran sobre todo a los estados del sureste de México (Ruiz et. al., 2012 y Moreno-Brid et. al., 2009).

Por la diferencia de tamaño de ambas economías, así como por la falta de fuentes de crecimiento internas en el caso de México, podemos deducir que existe causalidad del desempeño de la economía grande hacia la pequeña. Lo anterior se confirma con pruebas de causalidad de Granger, realizadas a nivel mensual y trimestral, con series desestacionalizadas de las producciones industriales de EE.UU. y México, entre 1980 y 2012 (Apéndice). Los fenómenos de interdependencia y dependencia entre economías ya habían sido señalados desde hace mucho tiempo en la literatura económica, resaltando sus aspectos macroeconómicos en un modelo keynesiano. Dorbusch (1980) es un excelente ejemplo de este tipo de literatura, en donde se observan efectos mucho mayores, favorables o desfavorables, dependiendo de la situación, de las economías grandes hacia las economías pequeñas.

Las figuras son importantes porque ilustran la estrecha relación entre las economías y, dada la causalidad de Granger, muestran cómo la actividad económica de EE.UU. afecta a la de México. Las pruebas de causalidad de Granger indican precedencia estadística, es decir, que un evento A ocurre previo a un evento B en el tiempo. Lo anterior significa estadísticamente que, cuando A se presenta, podemos esperar que B ocurra, aunque ello no signifique que B sea necesariamente causado por A. Sin embargo, en casos como el de la relación económica entre EE.UU. y México, su interpretación coincide con la idea que tenemos de causalidad en las ciencias duras o en las ciencias sociales (Hamilton, 1994).

Las 3 figuras que siguen muestran la relación, a nivel del crecimiento del PIB y de la evolución de la producción industrial y manufacturera, que existe entre las dos economías. Resalta en estas figuras que, a partir de la entrada en vigor del Tratado de Libre Comercio de América del Norte (TLCAN) en 1994, la relación se ha vuelto todavía más sólida.

En la figura 1 se observa que el crecimiento del PIB de EE.UU. tiene un efecto importante sobre el crecimiento del PIB de México, en especial a partir del TL- 
CAN, efecto que se transmite sobre todo a través de la producción industrial: la evolución de este sector de aquel país determina el comportamiento de la producción manufacturera e industrial mexicana, lo que se reporta en las figuras 2 y 3 . Calvo et. al. resaltan el ciclo de crecimiento (y tasas de interés) que la economía de EE.UU. induce no solamente en la economía mexicana sino en general en las economías emergentes (Calvo et. al., 2001).

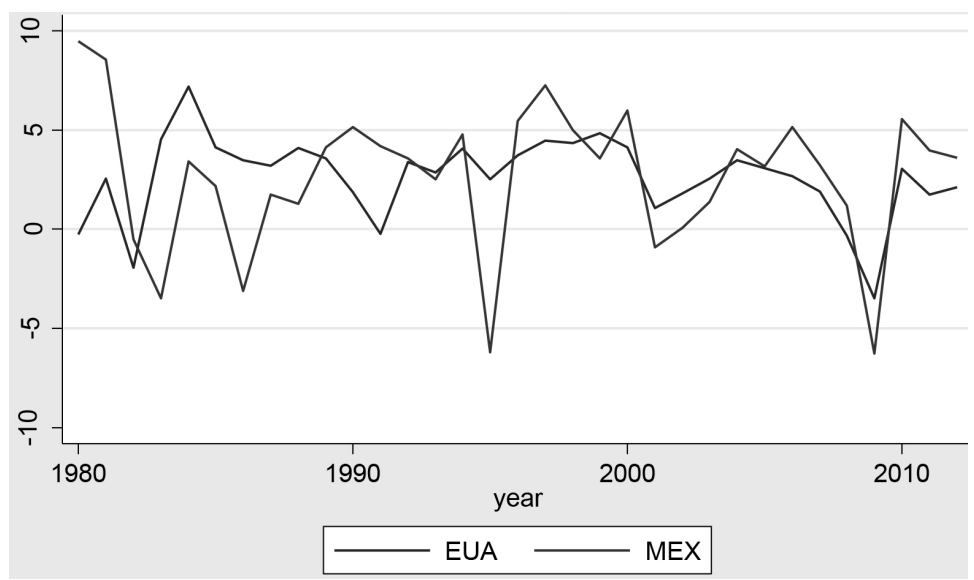

Figura 1. Tasa de crecimiento del PIB, México vs EUA, en términos reales.

Fuente: FMI, Panaroma Económico Mundial, abril de 2012.

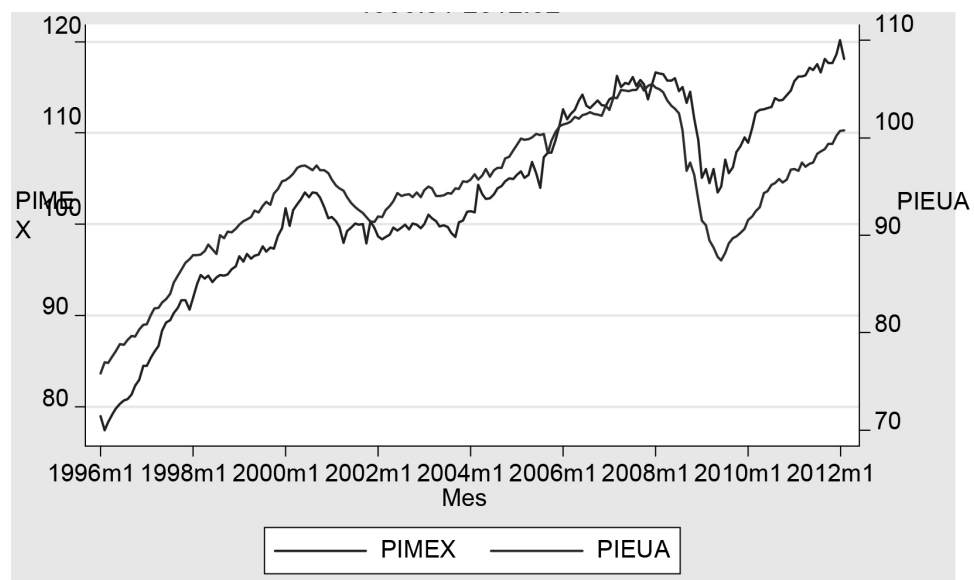

Figura 2. Producción industrial de México y de EUA, 1996:01-2012:02.

Fuente: BIE, Inegi, mayo de 2012. 


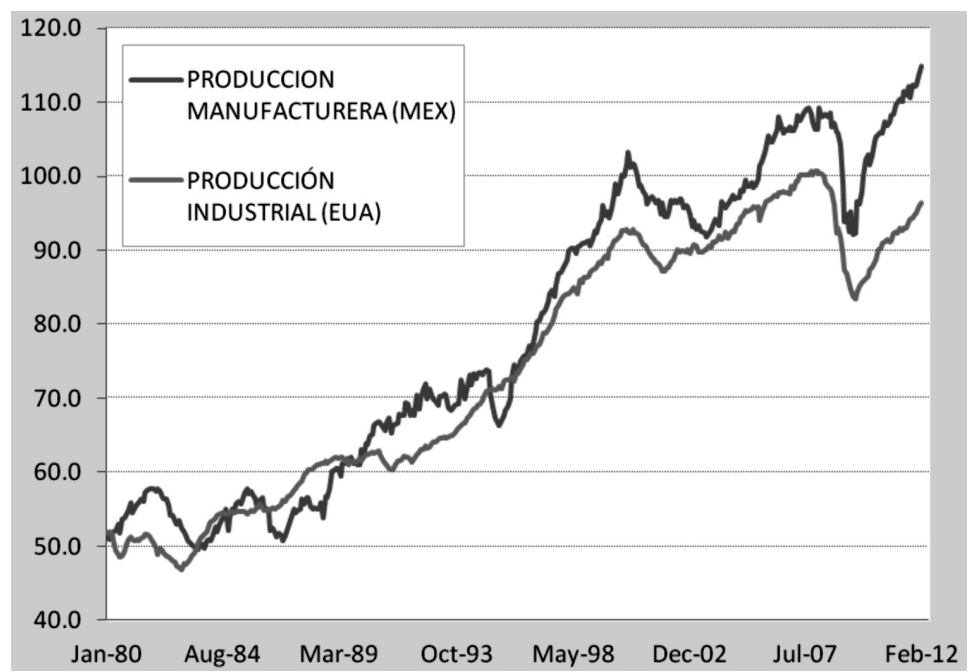

Figura 3. Producción manufacturera de México e industrial de EUA.

Fuente: OCDE y Junta de Gobernadores del Sistema de la Reserva Federal (FRED), mayo de 2012.

Como puede observarse en la figura 4, la interconexión industrial se manifiesta a través del comercio exterior entre México y EE.UU.. Con la evidencia de las pruebas de causalidad de Granger, podemos afirmar que las exportaciones de México dependen de las importaciones de EE.UU. En particular, la profunda contracción, cercana a 50\%, que registraron las importaciones de EE.UU. entre mediados de 2008 y principios de 2009 se reflejó en una contracción semejante, cercana a $40 \%$, en las exportaciones mexicanas en ese mismo periodo.

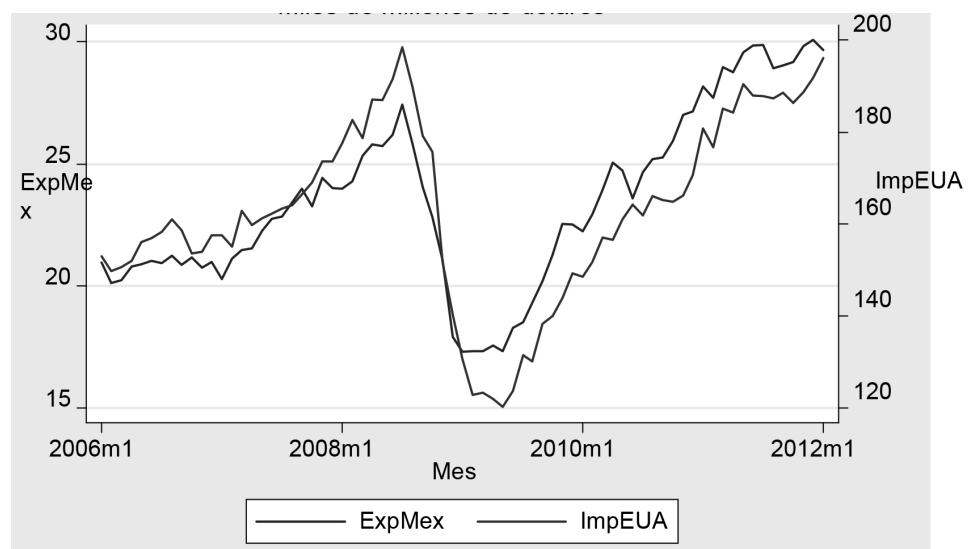

Figura 4. Exportaciones de México e Importaciones de EUA, miles de millones de dólares. Fuente: BIE, Inegi, mayo de 2012. 
Frente a la caída de la producción industrial de EE.UU. y la correspondiente contracción de las importaciones asociadas a dicho sector , las exportaciones mexicanas, que en cerca de $80 \%$ se destinan al mercado estadounidense, registraron una disminución importante. Asimismo, al recuperarse la producción industrial de nuestro principal socio comercial, también se recuperaron sus importaciones $\mathrm{y}$, por lo tanto, las exportaciones de México.

Aunque el mecanismo central de transmisión de la crisis financiera de 2008 hacia la economía mexicana fue el sector real, eso no significa que los mercados financieros del país no se ajustaran frente a este fenómeno. En particular, los precios de las acciones cotizadas en la Bolsa Mexicana de Valores exhibieron en promedio (el Índice de Precios y Cotizaciones, valuado en dólares) un comportamiento muy semejante al del Dow Jones, como se observa en la figura 5. La caída del índice mexicano fue incluso mayor, y su recuperación también. Entre 2008 y 2009, se contrajo cerca de 60\%, mientras que el Dow Jones lo hizo alrededor de 50\%. El índice mexicano, sin embargo, se recuperó a sus niveles previos a la caída a finales de 2011; por su lado, el Dow todavía se encontraba por debajo. A principios de 2012, ambos observaron de nuevo una caída, debido a la frágil situación financiera de algunos países de la Unión Europea.

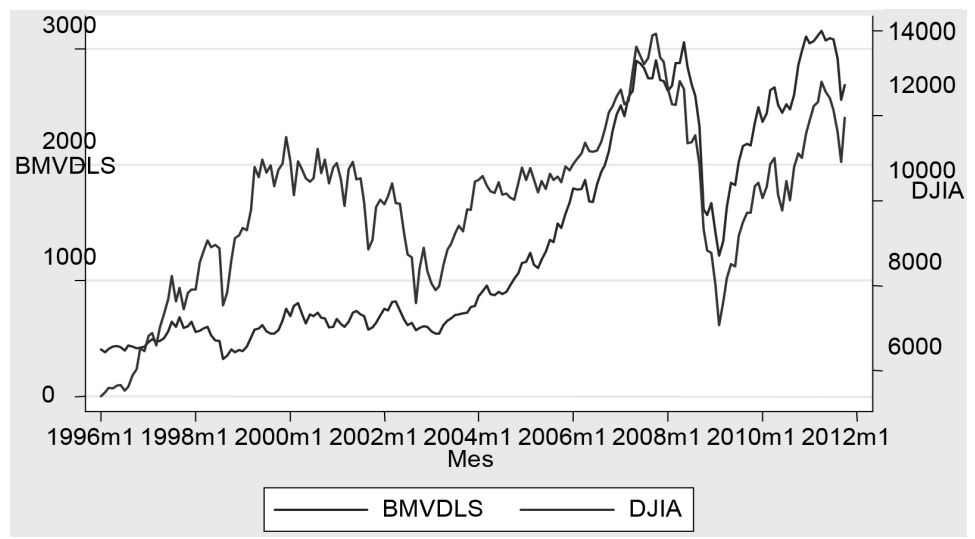

Figura 5. Bolsa Mexicana de Valores y Dow Jones, índice de mercados de valores.

Fuente: FRED, Federal Reserve Bank of Saint Louis, mayo 2012.

Dado el tamaño del mercado de valores mexicano, sus repercusiones sobre la economía no ocurren como en otros países, a través del efecto riqueza y la variación consecuente en el consumo, sino a través de modificaciones en el tipo de cambio y las tasas de interés. Más que causalidad de la Bolsa hacia estas variables, como ocurrió en 1987, las variaciones de ésta, del tipo de cambio y de las tasas de interés, ocu- 
rren como parte del ajuste de la economía mexicana frente a perturbaciones externas.

Si los bonos del exterior y, en general, los activos externos se encarecen, puede ocurrir una salida de capitales, que provoca la depreciación del tipo de cambio y un incremento de las tasas de interés domésticas, así como una caída de la bolsa de valores.

En el caso de la crisis financiera, el tipo de cambio ha registrado variaciones debido a la incierta situación internacional; primero, por la sustancial elevación de los precios de los bonos estadounidenses, y en segundo término, por la incertidumbre con respecto al destino del euro (fig. 6; SHCP, 2012).

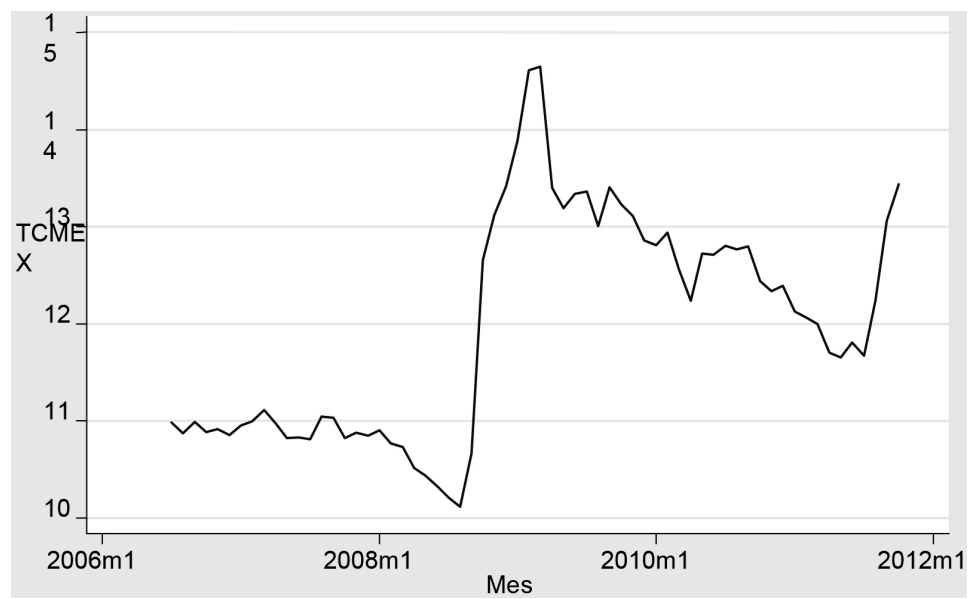

Figura 6. Tipo de cambio del peso con respecto al dólar.

Fuente: FRED, Federal Reserve Bank of Saint Louis, mayo 2012.

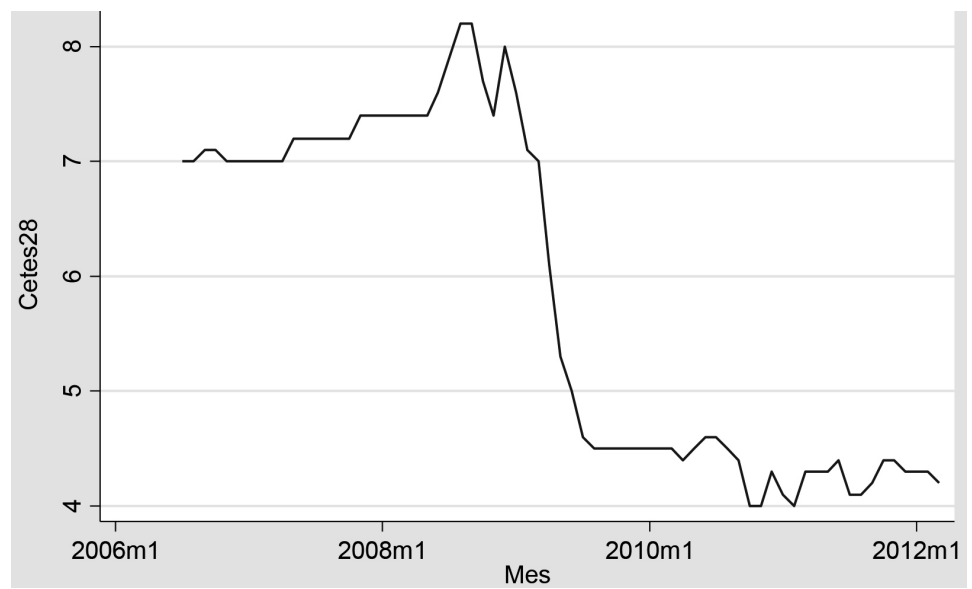

Figura 7. Tasa de interés de los Cetes a 28 días

Fuente: FRED, Federal Reserve Bank of Saint Louis, mayo de 2012. 
Las tasas de interés se han reducido aproximadamente en tres puntos porcentuales a partir de la crisis financiera (fig. 7 y 8); pese a su marcada caída en EE.UU., la percepción de riesgo a nivel internacional aumentó, contrarrestando ese efecto.

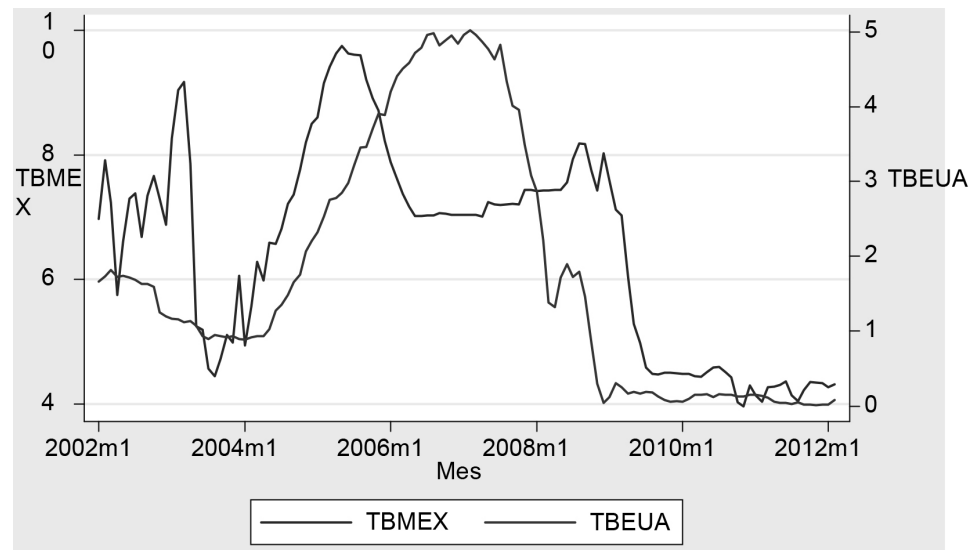

Figura 8. Tasa de interés (TBills) de México y EU, 2002:01-2012:02

Fuente: FRED, Federal Reserve Bank of Saint Louis, mayo de 2012.

\section{Algunos efectos regionales de la crisis de 2008}

En la figura 8 se aprecia que la contracción de la producción manufacturera producto de la crisis fue de alrededor de $30 \%$ en los casos de Baja California y Tamaulipas; por su parte, Sinaloa alcanzó aproximadamente 10\%. Se observa que el incremento en producción industrial del DF, logrado entre 2003 y 2008, se perdió con la crisis y no se ha recuperado aún. En contraste, Baja California ha repuntado de manera importante desde el segundo semestre de 2009 y se ubica muy cerca del mayor nivel que logró alcanzar en el periodo (entre 2007 y 2008). Tamaulipas, sin embargo, después de un repunte considerable, volvió a caer en forma notable y se ubica, a principios de 2012, en un nivel de producción industrial semejante a 2003.

La producción industrial mexicana asociada de forma más directa a la de EE.UU. se ubica en los estados de la frontera norte. Por ello, éstos sufrieron los mayores efectos de la crisis y se han ido recuperando conforme la economía de EE.UU. también se ha recuperado (Villarreal, 2010). En las siguientes 2 figuras se observa la evolución de la producción manufacturera de esos estados, contrastándola con la del Distrito Federal, entidad cuya actividad industrial se encuentra menos vinculada a la de EE.UU.

En la primera figura, se ubican estados que registran un comportamiento en forma de U invertida en la producción industrial entre 2003 y 2009, lo que significa que entre estos años prácticamente no avanzaron en dicho rubro. 


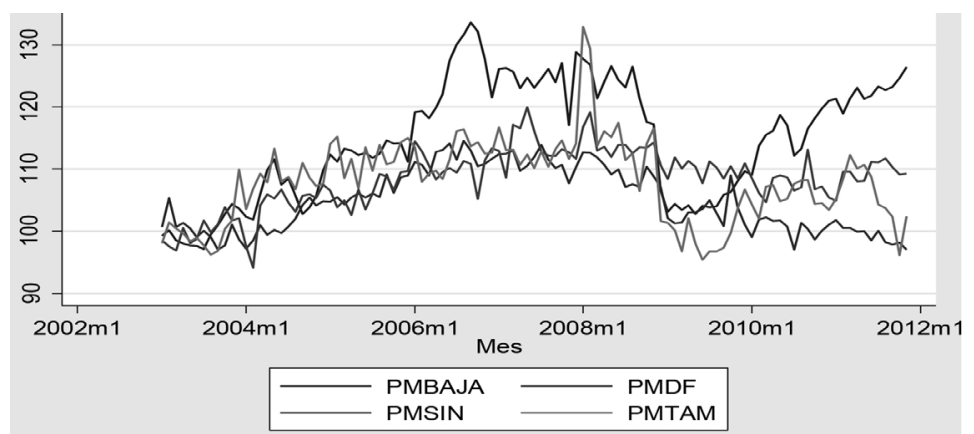

Figura 9. Producción manufacturera 2003-2011 (Baja California, Distrito Federal, Sinaloa y Tamaulipas). Fuente: BIE, Inegi, mayo de 2012.

Esto significa que en Tamaulipas y el Distrito Federal, la producción industrial per cápita cayó a la misma tasa que creció la población; en el caso de Sinaloa, ésta se mantuvo estancada, y solamente creció en Baja California, cerca de 1.3\% anual.

Los estados de Chihuahua, Coahuila, Nuevo León y Sonora, como se observa en la figura 10, han repuntado en su producción industrial después de la profunda contracción que registraron con la crisis. Coahuila y Sonora observaron caídas cercanas a 40\%, mientras que Nuevo León y Chihuahua, de alrededor de 30\%, con respecto a sus mayores niveles, alcanzados a mediados de 2008. En comparación con el año inicial (2003), los cuatro estados registran una producción industrial mayor a principios de 2012: Chihuahua, aproximadamente 15\%; Coahuila, 20\%; Nuevo León, 45\%, y Sonora, más de 50\%.

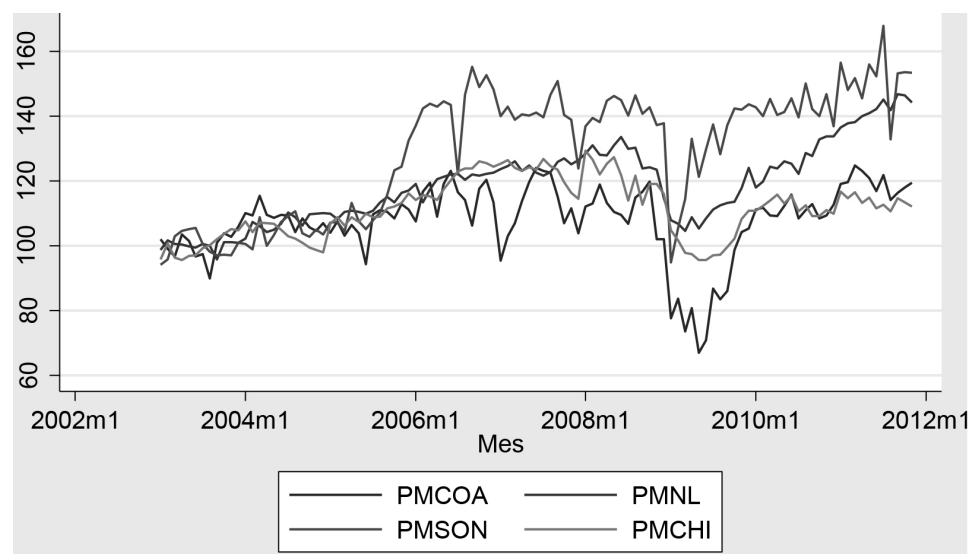

Figura 10. Producción manufacturera 2003-2011 (Chihuahua, Coahuila, Nuevo León y Sonora). Fuente: BIE, Inegi, mayo de 2012. 
El crecimiento promedio anual resulta raquítico en los casos de Coahuila y Chihuahua, 1.7 y $1.3 \%$, respectivamente, apenas por encima del crecimiento poblacional; en los casos de Nuevo León y Sonora, las tasas de crecimiento son de 3.4 y $4 \%$ de forma respectiva.

\section{Un modelo var de corto plazo para la producción manufacturera mexicana que incorpora el efecto de la producción industrial estadounidense}

Para trazar la interrelación de variables macroeconómicas mexicanas importantes con la producción industrial de EE.UU., se elaboró un modelo VAR mensual desestacionalizado (Baum, 2012), que contiene como variables endógenas, en ese orden, a la producción manufacturera, al tipo de cambio real, al gasto público real y a la oferta monetaria real. La única variable exógena es la producción industrial de EE.UU. Las variables se desestacionalizaron con el paquete estadístico TRAMO.

La muestra considerada abarca de mayo de 1986 a enero de $2012^{2}$. Se utilizaron tres rezagos de las variables endógenas de acuerdo con el criterio de Akaike (se probaron desde un rezago hasta 16) ${ }^{3}$. Todas las variables resultaron significativas al 1\%, de acuerdo con la prueba conjunta Chi cuadrada (Apéndice).

Las variables se obtuvieron de las siguientes fuentes: la producción manufacturera y el Índice Nacional de Precios al Consumidor, del Sistema de Información Económica del INEGI; la oferta monetaria (M2) y el tipo de cambio real del Banco de México, las cifras de gasto público del Sistema de Estadísticas Oportunas de Finanzas Públicas de la Secretaría de Hacienda y Crédito Público y, finalmente, los datos sobre la producción industrial de EE.UU. del Sistema FRED del Banco de la Reserva Federal de Saint Louis.

El modelo se corrió en tasas de crecimiento, ya que en niveles mostraba inestabilidad. En tasas de crecimiento, las raíces características de la matriz acompañante del modelo utilizado se ubican todas dentro del círculo unitario, lo que implica que dicho modelo es estable (Apéndice).

La idea es que, aparte de la producción industrial de EE.UU., la producción manufacturera se ve afectada por variables de demanda: por las políticas fiscal y monetaria, así como por el tipo de cambio real.

Ya que el determinante más importante de la producción manufacturera mexicana es la producción industrial estadounidense, en la descomposición de varianza resulta que solamente el tipo de cambio real tiene cierta importancia (explica cerca

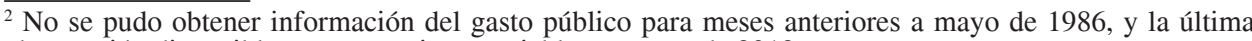
observación disponible para esta misma variable era enero de 2012.

${ }^{3}$ Los resultados fueron los mismos utilizando los criterios HQIC y SBIC.
} 
de 5\% de la varianza de la producción manufacturera), aparte de la propia producción manufacturera, como se observa en las 2 figuras siguientes.

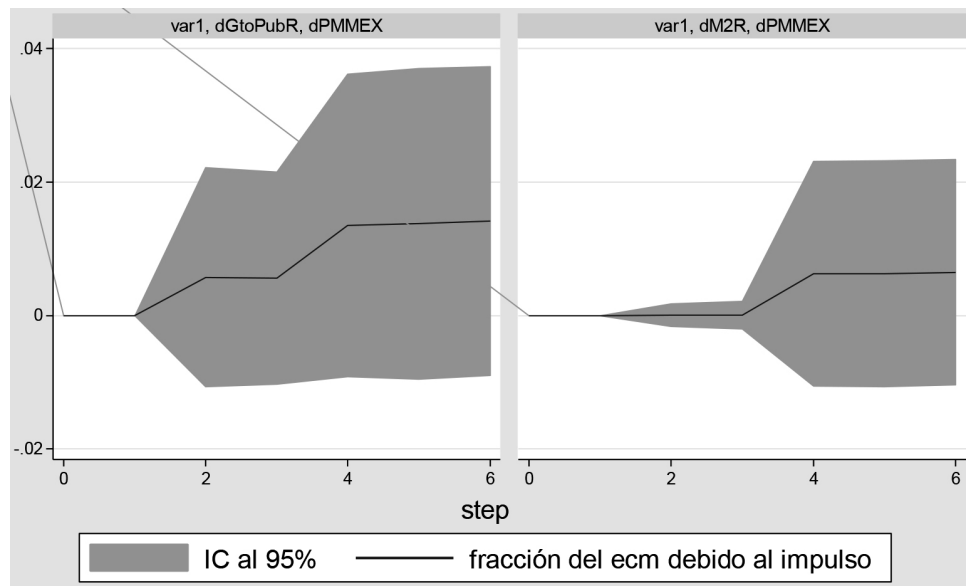

Figura 11. Descomposición de varianza de la producción manufacturera (Atribuible a GtoPubR y M2R). Figuras de la descomposición de varianza de la prod. man. en respuesta a GtoPubR y M2R.

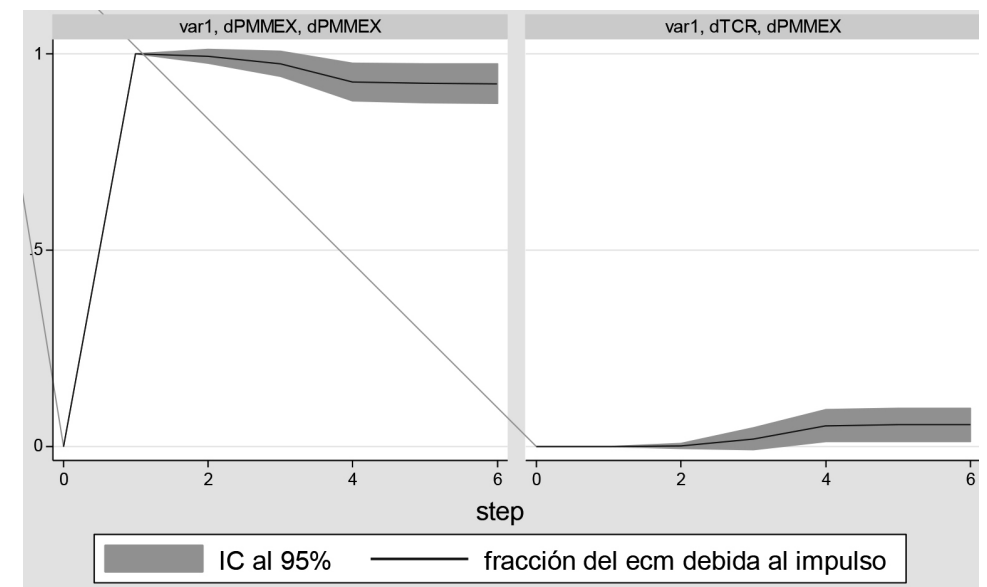

Figura 12. Descomposición de varianza de la producción manufacturera (Atribuible a la propia prod. man. y al TCR).

Figuras de la descomposición de varianza de la prod. man. en respuesta a la prod. man. y al TCR.

Las funciones de impulso-respuesta (o de respuesta al impulso), considerando perturbaciones a las variables de demanda que afectan la producción manufacturera, muestran que el tipo de cambio real tiene un efecto negativo de corto plazo sobre ésta (dicho resultado ya es conocido, Rodrik, 2008), básicamente porque en el 
caso de México las devaluaciones reales casi siempre han ocurrido, en la época moderna, en respuesta a choques externos negativos que han afectado de forma desfavorable la producción y el empleo.

Se observa también que las políticas monetaria y fiscal tienen un efecto positivo, aunque poco significativo, de corto plazo (el de la política fiscal se revierte casi de inmediato) sobre la producción manufacturera. La política fiscal tiene un efecto inmediato, mientras que la política monetaria registra cierto rezago: el shock a la tasa de crecimiento de la oferta monetaria real se transmite después de tres periodos a la tasa de crecimiento de la producción manufacturera. Nótese que estos resultados admiten la posibilidad de que las políticas monetaria y fiscal sean procíclicas en lugar de contracíclicas. Es decir que cuando la producción manufacturera se expanda, haya también expansión fiscal (aumentan los impuestos por la mayor actividad económica, lo que conduce a un aumento en el gasto público) y monetaria (la autoridad monetaria eleva la oferta monetaria al haber una mayor actividad económica que implica un incremento en la demanda de dinero).

Para poder contrarrestar, al menos en parte, los efectos negativos de perturbaciones externas, se requiere modificar esta situación: es decir, poder articular políticas fiscal y monetaria contracíclicas a partir del equilibrio fiscal intertemporal (no periodo a periodo) y de la estabilidad financiera (Frankel, J., C. Vegh y G. Vuletin, 2011; Jara et. al., 2009; así como Vegh, C. y G. Vuletin, 2012).

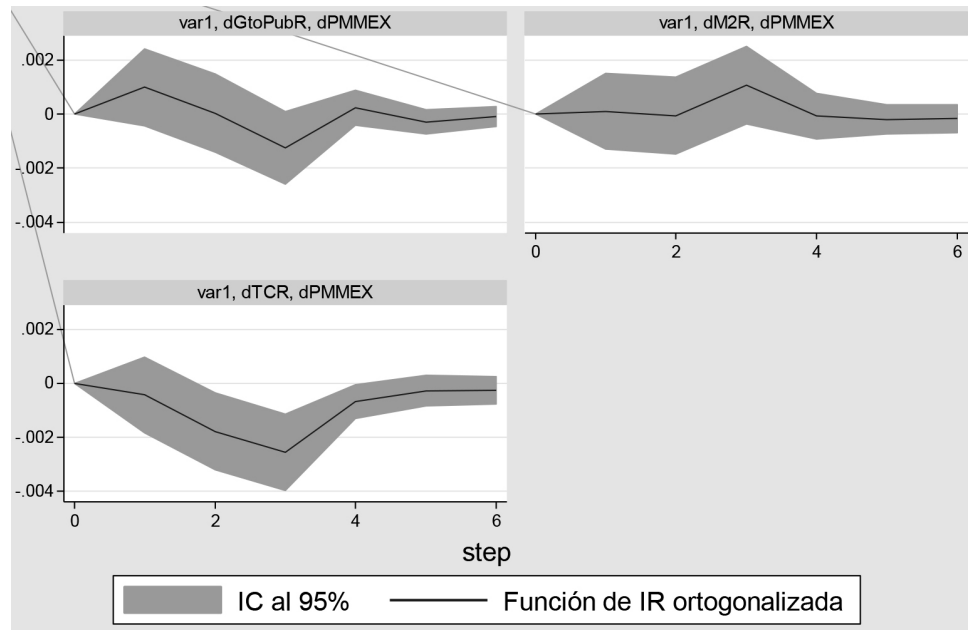

Figura 13. Respuesta de la TC de la prod. man. a shocks en TCR, GtoPubR y M2R.

Figuras de respuesta a la prod. man. 


\section{Conclusiones}

La crisis financiera y, posteriormente, económica de 2008, cuyo origen se ubica en EE.UU. y, en particular, en las hipotecas denominadas supbrime, ha conducido a la contracción mundial de la actividad económica y el empleo, así como a un aumento en la percepción de riesgo por parte de los inversionistas a nivel internacional.

En el caso de México, el mecanismo de transmisión de esta crisis, al menos hasta 2012, es la producción industrial, en particular, la manufacturera, asociada al comercio con nuestro más importante socio, EE.UU. Es decir, el mecanismo de transmisión de más relevancia fue el sector real y no el financiero, como en otros países.

En el corto plazo, esto produjo una contracción importante de la actividad económica nacional, al disminuir de manera significativa el comercio con EE.UU. Los efectos nocivos de esto se observaron de forma específica en la región norte del país, cuya evolución económica está profundamente vinculada a la de nuestro vecino del norte. Al recuperarse la producción industrial de éste, también se recuperó la actividad económica de dicha región, aunque es difícil cantar victoria frente a la incertidumbre que todavía prevalece en la economía mundial.

¿Qué puede hacer una economía pequeña y abierta como la nuestra frente a esto? ¿Continuar dependiendo tan drásticamente del destino de la economía de EE.UU? Por supuesto que, al menos en el mediano plazo, la dependencia continuará siendo significativa, pero es fundamental desarrollar fuentes internas de crecimiento si queremos tener cierta independencia en el crecimiento económico. Asimismo, la recuperación de condiciones de normalidad en el sistema financiero internacional sin duda afectarán los flujos de capital hacia las economías emergentes, sus tipos de cambio y sus tasas de interés. De hecho, la incertidumbre acerca de cuando ocurrirá tal normalización ya ha causado efectos sobre todo en el tipo de cambio y en el grado de riesgo de muchas economías emergentes, sobre todo aquellas cuyas condiciones económicas y financieras son frágiles.

Es importante también tener la capacidad de llevar a cabo políticas de demanda que contrarresten, al menos en parte, los efectos nocivos de las perturbaciones externas, en especial si son tan virulentas como la actual. Para ello, se requiere tener finanzas públicas intertemporalmente sanas, es decir, no es necesario tener equilibrio fiscal cada año, pero sí a través del ciclo económico, así como se requiere estabilidad financiera que permita utilizar la política monetaria para atenuar los efectos nocivos de los choques externos, a través de la reducción de las tasas de interés domésticas y de la disponibilidad de crédito del banco central a las instituciones financieras del país a un costo razonable. 


\section{Apéndice}

Pruebas de causalidad mensual y trimestral de la producción industrial de EUA (PIEUA) versus la producción manufacturera (PMANMEX) de México: 1980-2012.

Prueba mensual:

Date: 05/29/12 Time: 15:34

Sample: 1980M01 2012M01

Lags: 6

\begin{tabular}{lccc}
\hline Null Hypothesis: & Obs & F-Statistic & Prob. \\
\hline PMANMEX does not Granger Cause PIEUA & 379 & 1.09255 & 0.3662 \\
PIEUA does not Granger Cause PMANMEX & & 5.43431 & 2.E-05 \\
\hline
\end{tabular}

Prueba trimestral:

Pairwise Granger Causality Tests

Date: 05/29/12 Time: 16:01

Sample: 1980Q1 2011Q4

Lags: 2

\begin{tabular}{lccc}
\hline Null Hypothesis: & Obs & F-Statistic & Prob. \\
\hline PIEUA does not Granger Cause PMANMEX & 126 & 9.17201 & 0.0002 \\
PMANMEX does not Granger Cause PIEUA & & 0.88767 & 0.4143 \\
\hline
\end{tabular}

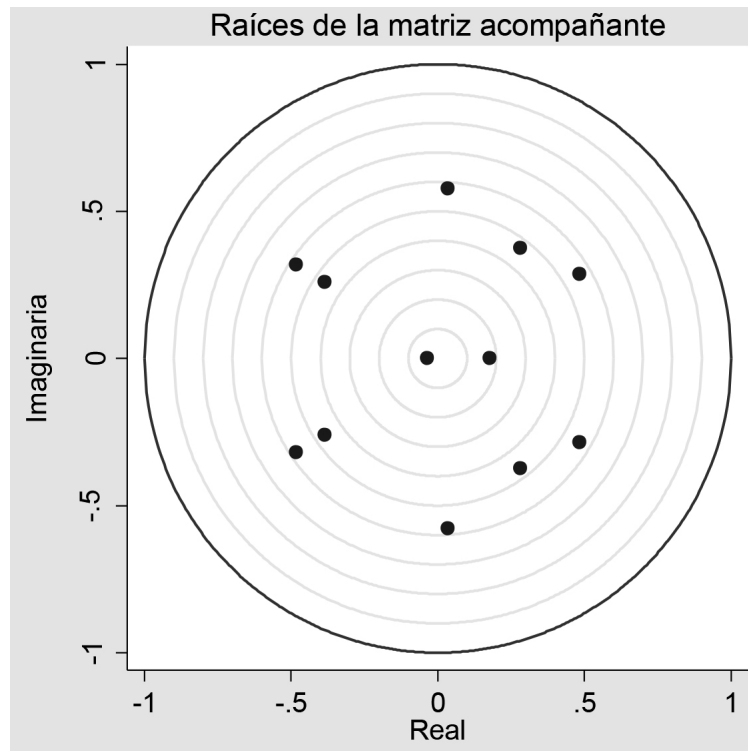


Las raíces características de la matriz acompañante se encuentran dentro del círculo unitario, por lo que el VAR satisface la condición de estabilidad.

Los resultados del modelo VAR utilizado, de acuerdo con el reporte del paquete econométrico STATA, se presentan a continuación:

. var dPMMEX dTCR dM2R DGtoPubR, lags (113) exog(dPIEUA L.dPIEUA L2. dPIEUA)

Sample: $1986 \mathrm{~m} 5-2012 \mathrm{~m} 1$

Log likelihood $=2099.2$

FPE $\quad=2.24 \mathrm{e}-11$

Det $($ Sigma_ml $)=1.48 \mathrm{e}-11$

No. de obs. $=309$

AIC $=-13.2$

$\mathrm{HQIC}=-12.9$

$\mathrm{SBIC}=-12.4$

\begin{tabular}{lccccc}
\hline Equation & Parms & RMSE & R-Sq & Chi2 & P>Chi2 \\
\hline dPMMEX & 16 & 0.013 & 0.205 & 79.6 & 0.0000 \\
dTCR & 16 & 0.033 & 0.125 & 44.2 & 0.0001 \\
dM2R & 16 & 0.016 & 0.091 & 30.8 & 0.0093 \\
dGtoPubR & 16 & 0.702 & 0.180 & 67.9 & 0.0000 \\
\hline
\end{tabular}

\section{Referencias}

Banco de Información Económica (BIE), INEGI, Disponible en: http://www.inegi.org.mx/sistemas/ bie, fecha de consulta 21 de mayo de 2012.

Banco de México, Informe Anual 2008.

Banco de la Reserva Federal de Saint Louis, Sistema de Información FRED, Disponible en: https:// research.stlouisfed.org/fred2/, fecha de consulta 20 de mayo de 2012.

Baum, C. F. (2006). An Introduction to Modern Econometrics Using Stata. Stata Press. Estados Unidos.

Calvo, G., E. Fernández, C. Reinhart y E. Talvi (2001). The Growth-Interest-Rate Cycle in the United States and Its Consequences for Emerging Markets. Documento de trabajo no. 385 del Banco Interamericano de Desarrollo.

CEPAL (2009), Panorama de la inserción internacional de América Latina y el Caribe 2008-2009: Crisis y espacios de cooperación regional.

Chinn, M. D. (2012). Evidence on Financial Globalization and Crises: Global Imbalances. En G.Caprio (Ed.), Encyclopedia of Financial Globalization. Elsevier.

Dornbusch, R. (1980). Open Economy Macroeconomics. Basic Books Inc. Publishers. Estados Unidos.

Eichengreen, B. (2010). Lessons of the crisis for emerging markets. International Economics and Economic Policy, (7), 49-62.

Fondo Monetario Internacional (FMI) (2012), Base de datos del Panorama Económico Mundial, Estados Unidos. 
Frankel, J., C. Vegh y G. Vuletin (2011). Fiscal policy in developing countries: Escape from procyclicality. Disponible en: http://voxeu.org/article/how-developing-nations-escaped-procyclical-fiscal-policy, fecha de consulta julio 10 de 2012.

Hamilton, J. D. (1994). Time Series Analysis. Princeton University Press. Estados Unidos.

Hernández, F. (2010). La crisis financiera y su impacto en México. Disponible en: http://www.academia.edu/12342395/La_crisis_financiera_y_su_impacto_en_México, fecha de consulta agosto 15 de 2012.

Jara, A., R. Moreno y C. E. Tovar (2009). La crisis internacional y América Latina: repercusiones financieras y políticas de respuesta. Informe Trimestral del Banco de Pagos Internacionales, segundo trimestre. Suiza.

Lane, P. R. (2013). Financial Globalisation and the Crisis. Open Economy Review, (24), 555-580.

Moreno Brid, J. C. e I. Paunovic (2009). Crisis financiera internacional y sus efectos en la economía mexicana. Economía: Teoría y Práctica, (1), 63-80.

Nayak, S. (2013). The Global Financial Crisis: Genesis, Policy Response and Road Ahead. Springer. Suiza.

Ocampo, J. A. (2009). Impactos de la crisis financiera mundial sobre América Latina. Revista Cepal, (97), 9-32.

OCDE (2012), Estadísticas de producción incustria. Disponible en: stats.oecd.org/index.aspx?query$\mathrm{id}=207$, fecha de consulta 16 de junio de 2012.

Rodríguez, J. F. y J. Zurita (2008), La crisis de los créditos hipotecarios en Estados Unidos: algunas consideraciones, Análisis Económico, 23 (54), 331-348.

Rodrik, D. (2008). The Real Exchange Rate and Economic Growth. Disponible en: http://www.hks. harvard.edu/fs/drodrik/Research\%20papers/RER\%20and\%20growth.pdf, fecha de consulta 20 de abril de 2008.

Ruiz, I. y C. Vargas (2012). Exploring the causes of the slowdown in remittances to Mexico. Empirical Economics, (42), 745-766.

SHCP (2012). Memoria 2006-2012. Disponible en: http://www.shcp.gob.mx/LaSHCP/Publicaciones/credito/memorias_shcp_2006_2012.pdf, fecha de consulta 14 de septiembre de 2012.

Tanzi, V. (2011). La crisis financiera y económica de 2008-2009: Efectos fiscales y monetarios. Disponible en: http://www10.iadb.org/intal/intalcdi/PE/2011/08050.pdf, fecha de consulta 15 de julio de 2012.

Vegh, C. y G. Vuletin (2012). Graduation from monetary policy procyclicality. Disponible en: http:// www.voxeu.org/article/graduation-monetary-policy-procyclicality, fecha de consulta 10 de septiembre de 2012.

Vidal, G., W. C. Marshall y E. Correa (2011). Differing Effects of the Global Financial Crisis: Why Mexico Has Been Harder Hit Than Other Large Latin American Countries. Bulletin of Latin American Research, 30 (4), 419-437.

Villarreal, M. A. (2010), The Mexican Economy After the Global Financial Crisis. Congressional Research Service. Disponible en: http://fpc.state.gov/documents/organization/148789.pdf, fecha de consulta 18 de mayo de 2012. 